\title{
Cystoid macular edema associated with preservative-free latanoprost after uncomplicated cataract surgery: case report and review of the literature
}

\author{
Olga E. Makri', Foteini N. Tsapardoni ${ }^{1}$, Panagiotis Plotas ${ }^{1}$, Nikistratos Ifantis ${ }^{1}$, Paraskevi T. Xanthopoulou ${ }^{2}$ \\ and Constantine D. Georgakopoulos ${ }^{1 *}$ (D)
}

\begin{abstract}
Background: Cystoid macular edema associated with latanoprost administration has been reported in patients after complicated cataract surgery with coexisting risk factors. We present the first case of preservative free latanoprost associated cystoid macular edema that occurred many months after uncomplicated cataract surgery.

Case presentation: A 65-year old Caucasian female presented in the Outpatients Clinic complaining of reduced vision and metamorphopsia in the right eye. She had undergone uneventful phacoemulsification 19 months ago in the right eye and was under treatment with preservative free latanoprost eye drops for the last 7 months for ocular hypertension. Her remaining medical and ocular history were otherwise unremarkable. Cystoid macular edema with serous retinal detachment was diagnosed in the right eye using optical coherence tomography and fluorescein angiography. Latanoprost was discontinued and brinzolamide and nepafenac eye drops were administered in the right eye. Two months later, cystoid macular edema completely resolved with restoration of visual acuity. Nepafenac eye drops were administered for another 2 months. Eight months after latanoprost cessation optical coherence demonstrated no sign of cystoid macular edema whereas a subtle epiretinal membrane was noted.

Conclusions: Cystoid macular edema may potentially occur in patients receiving preservative free latanoprost. More interestingly, in our case it was diagnosed in a patient with a long standing pseudophakia after uncomplicated phacoemulsification. No obvious risk factor for macular edema development was recognized. Prompt diagnosis and latanoprost discontinuation resulted in complete resolution of the cystoid macular edema and functional restoration of the eye.
\end{abstract}

Keywords: Cystoid macular edema, Latanoprost, Preservative free, Uncomplicated phacoemulsification

\section{Background}

Latanoprost (LP) is a phenyl-substituted prostaglandin analogue (PGA) that has become the most popular antiglaucoma medication for the treatment of primary open-angle glaucoma and ocular hypertension. Administered topically, LP lowers the intraocular pressure (IOP)

\footnotetext{
*Correspondence: cgeorg@upatras.gr

1 Department of Ophthalmology, Medical School, University of Patras, 26504 Patras, Greece

Full list of author information is available at the end of the article
}

presumably by increasing the uveoscleral outflow. The adverse effects of LP have been relatively mild and topical, mainly consisting of hypertrichosis, increased eyelash pigmentation, topical irritation, conjunctival hyperemia, superficial punctate keratopathy, and increased iris pigmentation while cystoid macular edema (CME) is considered a rare adverse effect [1].

There are several case reports presenting CME in early postoperative pseudophakias in eyes that are administered topical PGAs like LP, travoprost, bimatoprost, or unoprostone [2]. In the majority of these cases widely 
accepted risk factors that may potentially alter the blood-retinal barrier (BRB) and increase the risk of CME coexisted. These conditions include aphakia, complicated cataract surgery, vitreous loss, excessive intraoperative manipulations such as mechanical pupil stretch or iris prolapse during surgery, absent or ruptured posterior capsule, the presence of an anterior chamber intraocular lens, filtering or other glaucoma operations and intraocular surgeries, epiretinal membrane, history of uveitis or prior CME, retinal inflammatory or vascular disease like diabetes mellitus etc. [3, 4].

Latanoprost-related CME after uncomplicated phacoemulsification in absence of any systemic or ocular risk factors for developing CME is uncommon. There are reports of CME in six eyes treated with LP where the only recognized risk factor was previous uncomplicated cataract surgery [3]. However, details about these cases are lacking and, as the authors state, risk factors may be underestimated since five of them were spontaneous reports and one occurred during a phase III clinical trial. Yeh et al. report four cases where CME was clinically diagnosed 1 month after uneventful phacoemulsification, while LP was not discontinued perioperatively [4]. Thereafter, ten cases of PGA associated CME after uneventful cataract surgery in patients without known risk factors are described in more detail (Table 1) [5-11].

In the following report we describe a patient with a history of uncomplicated phacoemulsification surgery 19 months ago who developed CME 7 months after initiation of preservative free LP.

\section{Case presentation}

A 65-year old Caucasian female presented in the Outpatients Clinic complaining of gradually reduced vision and metamorphopsia in the right eye (OD). Patient's medical and ocular history were unremarkable. She had undergone uncomplicated cataract surgery with posterior chamber intraocular lens (IOL) implantation in OD 19 months ago and was on treatment with preservative free LP $0.005 \%$ (Monoprost $50 \mu \mathrm{g} / \mathrm{ml}$, Thea, ClermontFerrand, France) every night in both eyes (OU) for the last 7 months due to ocular hypertension. At the time that LP ophthalmic solution was initiated patient's best-corrected visual acuity (BCVA) was 20/20 OU and intraocular pressure (IOP) was $24 \mathrm{~mm} \mathrm{Hg}$ OU while Optical Coherence Tomography (OCT), conducted as baseline examination, was normal (Fig. 1a).

On admission, ocular examination disclosed BCVA $20 / 30$ in OD and 20/20 in OS. Slit lamp examination of OD revealed IOL in the capsular bag with intact posterior capsule with no evidence of intraocular inflammation or any other pathology such as ruptured posterior capsule. Slit lamp examination of OS was normal. Intraocular pressure was $14 \mathrm{mmHg}$ OU. Fundus examination revealed a slight decrease in the foveal reflectivity $\mathrm{OD}$ indicative of CME whereas no pathology was observed in OS. Optical coherence tomography in OD confirmed the diagnosis of CME demonstrating welldefined, intraretinal cystic areas of low reflectivity in the macula, mainly in the outer plexiform and inner nuclear layers of the retina, with serous retinal detachment (SRD) (Fig. 1b). Fluorescein angiography (FA) in OD revealed a petaloid leakage from the perifoveal retinal capillaries during the late phases without staining of the optic disc, while multiple areas of subtle fluorescein leakage was noted throughout the retina (Fig. 2a). No leakage or any other pathology was observed in the OS (Fig. 2b).

Latanoprost was discontinued and was replaced with brinzolamide eye drops $1 \%$ b.i.d (Azopt $10 \mathrm{mg} / \mathrm{ml}$, Alcon Laboratories Ltd., Hertfordshire, UK) OU. Nepafenac eye drops (Nevanac, $1 \mathrm{mg} / \mathrm{ml}$, Alcon Laboratories Ltd., Hertfordshire, UK) t.i.d. were administered in OD. One month later, BCVA was restored to $20 / 20$ in OD, metamorphopsia disappeared, and OCT demonstrated subsidence of $\mathrm{CME}$ with a single remaining intraretinal cyst. Two months after LP discontinuation, OCT demonstrated complete resolution of CME whereas a subtle epiretinal membrane was noted (Fig. 1c). At that time BCVA was 20/20 OU and IOP $17 \mathrm{mmHg}$ in OD and $18 \mathrm{mmHg}$ in OS. Nepafenac eye drops were administered for additional 2 months. Patient was examined every month thereafter. Eight months after LP cessation patient had BCVA 20/20 OU and IOP $18 \mathrm{mmHg}$ OU, while she remained on brinzolamide eye drops b.i.d. OU. Optical coherence tomography demonstrated no sign of CME.

\section{Discussion}

We describe the occurrence of CME with SRD in a pseudophakic patient 7 months after LP initiation and 19 months after uneventful phacoemulsification cataract extraction with posterior chamber IOL implant and intact posterior capsule. The diagnosis of CME was established with the characteristic OCT and FA findings, whereas no other possible causes of CME could be recognized in that eye. Although the patient was pseudophakic in OD for 19 months, there was no evidence of pseudophakic CME before LP initiation as it was indicated by clinical examination and a reference OCT, while CME was developed only in the pseudophakic eye treated with LP. Cystoid macular edema resolved with LP discontinuation and administration of nepafenac eye drops.

The exact mechanism by which LP may cause CME remains unclear. While LP does not seem to exhibit direct vasoactive or inflammatory properties, it is assumed that in early postoperative pseudophakias, LP affects the wound healing process of lens epithelial cells 


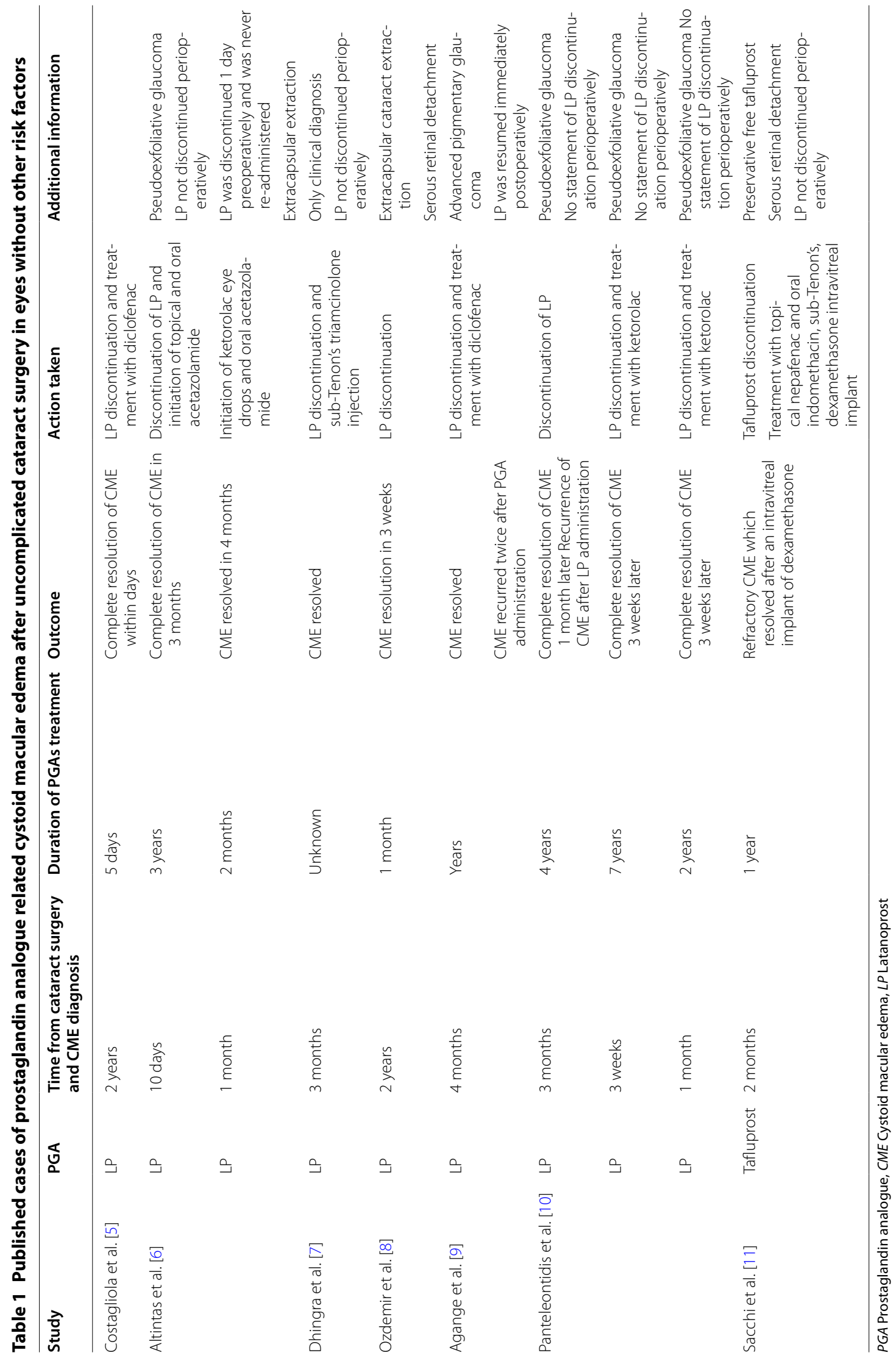



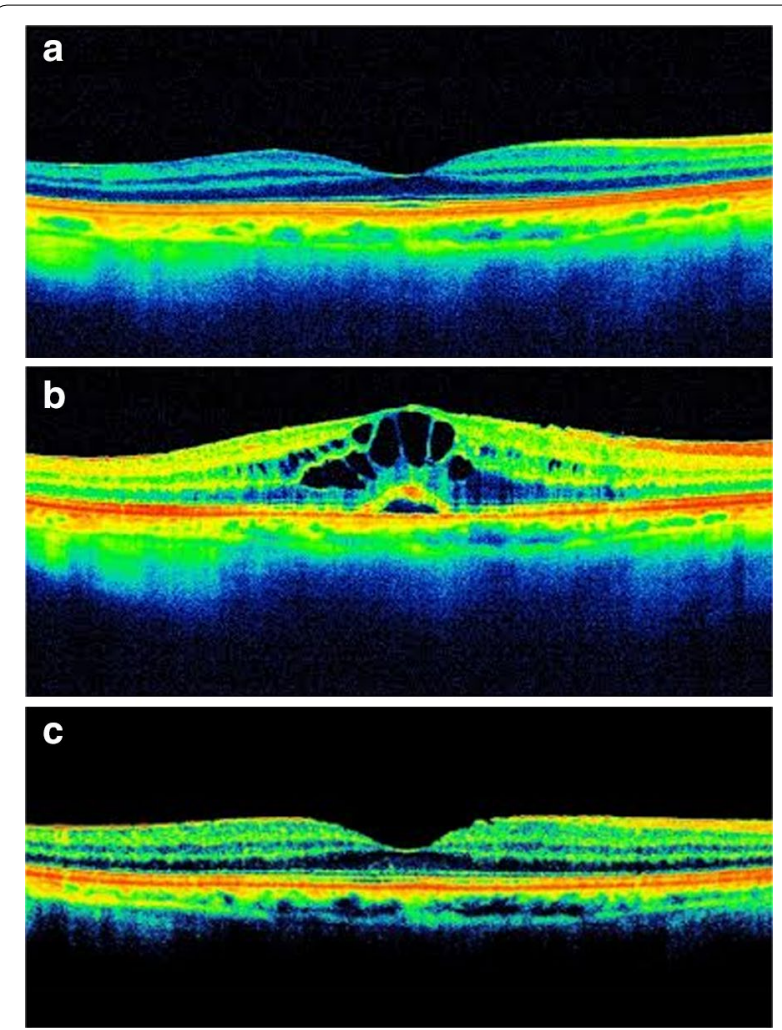

Fig. 1 a Optical coherence tomography of right eye before latanoprost administration. No pathology is detected. b Seven months after treatment with preservative free latanoprost optical coherence tomography in right eye revealed cystoid macular edema with well-defined, intraretinal cystic areas of low reflectivity in the macula with serous retinal detachment. c Two months after latanoprost discontinuation optical coherence tomography demonstrated complete resolution of cystoid macular edema. A subtle epiretinal membrane is noted

accelerating the biosynthesis of endogenous prostaglandins and other inflammatory mediators that eventually enhance disruption the blood-aqueous-barrier resulting in increase of incidence of angiographic CME [3, 12]. It is proposed that several risk factors such as complicated cataract surgery, vitreous loss, absent or ruptured posterior capsule render some eyes more prone to develop PG related CME [3, 4]. However, the vast majority of pseudophakic eyes treated with PGAs, even those with additional risk factors, do not develop CME [3]. Interestingly, our case does not have an obvious risk factor for developing CME except from the pseudophakic status itself.

Reviewing the cases in Table 1 , four cases have a common feature which is pseudoexfoliative glaucoma $[6,10]$. Pseudoexfoliation could be recognized as an independent risk factor since it results in abnormal blood-aqueous barrier predisposing to CME after phacoemulsification [13]. In two other cases CME occurred after uncomplicated extracapsular cataract extraction $[6,8]$. In these cases someone could argue that the intraoperative manipulations during extracapsular cataract extraction could pose a risk factor that could interfere with the BRB integrity [14]. Of the remaining cases there are three cases of PGA associated CME where PGA had been already administered before surgery without being discontinued perioperatively and during the early postoperative period when the BRB is unstable $[7,9,11]$.

In our case and in the case reported by Costagliola et al. [5] the CME occurred in eyes with long standing pseudophakias. In the case reported by Costagliola et al. [5] LP was prescribed 24-month after phacoemulsification and CME was diagnosed 5 days after initiation of LP treatment. Our patient had never received any antiglaucoma treatment until 12 months after surgery. While one could expect restoration of BRB in eyes with long standing pseudophakias, these two cases imply that bloodocular barrier might remain fragile to LP several months after uncomplicated phacoemulsification even in eyes without other risk factors for BRB disruption.

Interestingly, it has been proposed that the main cause of CME in early postoperative pseudophakias is not LP, but rather the benzalkonium chloride, added as preservative in many antiglaucoma eye drops [15]. Our case is the first reported case of CME associated with preservative free LP and in combination with the case reported by Sacchi et al. weakens the theory of preservative-induced CME [11].

\section{Conclusions}

We report a case of CME with SRD associated with LP administration with some interesting features. This is the first report of preservative free LP-induced CME. Furthermore, the CME occurred in an eye with a long standing pseudophakia after uncomplicated phacoemulsification. In our case no significant risk factor for CME development was recognized. Although the evidence for a causal association might be questioned and would be even more robust if CME recurred with rechallenge with LP, balancing the risk-benefit ratio we felt that it was unethical to rechallenge our patient in this setting because of the possibility of permanent visual loss [16]. The CME and SRD responded promptly to LP discontinuation and nepafenac administration. This temporal association suggests a causal relationship between topical application of LP and CME in our patient. Interestingly, the presence of pre-treatment normal OCT enhances a causal relationship.

There is not a single medication or surgical treatment without potential adverse effects and complications. Our case indicates the possibility of unexpected events related 

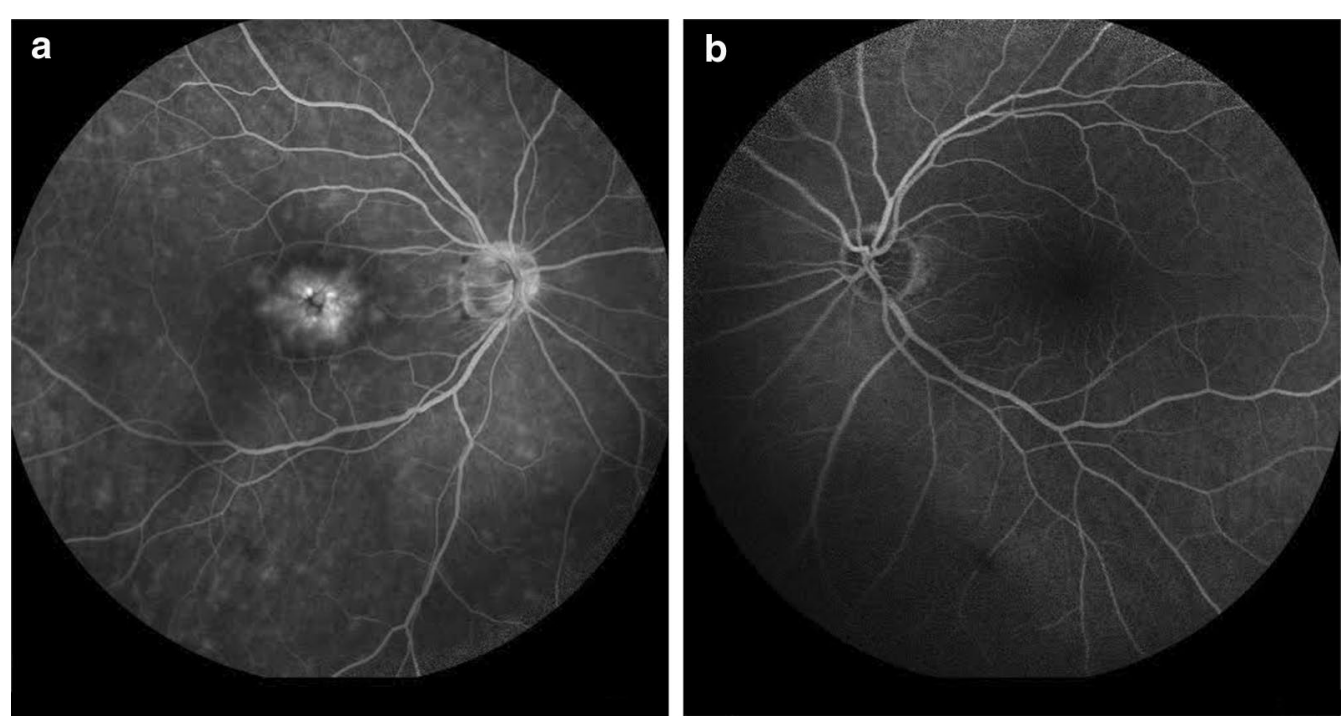

Fig. 2 a Fluorescein angiography in the right eye revealed a petaloid leakage from the perifoveal retinal capillaries during the late phases without staining of the optic disc. Multiple areas of subtle fluorescein leakage were noted throughout the retina. $\mathbf{b}$ No leakage or any other pathology was observed in the left eye

to LP administration after uncomplicated cataract surgery even in patients without any apparent risk factors.

\section{Abbreviations}

BCVA: best-corrected visual acuity; BRB: blood-retinal barrier; CME: cystoid macular edema; FA: fluorescein angiography; IOL: intraocular lens; IOP: intraocular pressure; LP: latanoprost; OCT: optical coherence tomography; OD: right eye; OS: left eye; OU: both eyes; PGA: prostaglandine analogue; SRD: serous retinal detachment.

\section{Authors' contributions}

OM participated in diagnosis and treatment of the patient, drafted the manuscript, collected the data and reviewed the literature. FT helped to draft the manuscript and participated in the literature search and information gathering. PP helped to draft the manuscript and participated in the literature search and information gathering. NI helped to draft the manuscript and participated in the literature search and information gathering. PX helped to draft the manuscript and participated in the literature search and information gathering. CG participated in diagnosis and treatment of the patient, drafted the manuscript, reviewed the literaturea and critically revised the manuscript for important intellectual content. All authors read and approved the final manuscript.

\section{Author details}

${ }^{1}$ Department of Ophthalmology, Medical School, University of Patras, 265 04 Patras, Greece. ${ }^{2}$ Department of Ophthalmology, 401 General Military Hospital of Athens, Athens, Greece.

\section{Acknowledgements}

We do not have anyone to acknowledge to for this case report.

\section{Competing interests}

The authors declare that they have no competing interests.

\section{Availability of data and materials}

All the data supporting our findings is contained within the manuscript.

\section{Consent for publication}

Written informed consent was obtained from the patient for publication of this Case Report and any accompanying images.

\section{Ethics approval and consent to participate}

The approval and consent to participate was not required as the data of the case report has been analyzed in a retrospective manner and has no effect on treatment of the patient.

Received: 21 October 2016 Accepted: 8 March 2017

Published online: 20 March 2017

\section{References}

1. Latanoprost summary of product characteristics. https://www.medicines. org.uk/emc/medicine/30599. Accessed 13 May 2016

2. Makri OE, Georgalas I, Georgakopoulos CD. Drug-induced macular edema. Drugs. 2013;73:789-802.

3. Schumer RA, Camras CB, Mandahl AK. Putative side effects of prostaglandin analogs. Surv Ophthalmol. 2002;47:S219.

4. Yeh PC, Ramanathan S. Latanoprost and clinically significant cystoid macular edema after uneventful phacoemulsification with intraocular lens implantation. J Cataract Refract Surg. 2002;28:1814-8.

5. Costagliola C, Del Prete A, Verolino M, Antinozzi P, Fusco R, Parmeggiani F, Mastropasqua L. Effect of $0.005 \%$ latanoprost once daily on intraocular pressure in glaucomatous patients not adequately controlled by betablockers twice daily: a 3-year follow-up. Experience and incidence of side effects in a prospective study on 76 patients. Graefes Arch Clin Exp Ophthalmol. 2002;240:379-86.

6. Altintaş O, Yüksel N, Karabaş VL, Demirci G. Cystoid macular edema associated with latanoprost after uncomplicated cataract surgery. Eur J Ophthalmol. 2005;15:158-61.

7. Dhingra N, Morgan JE. Pseudophakic cystoid macular oedema: 30 months after latanoprost challenge. Eye (Lond). 2007:21:269-71.

8. Ozdemir H, Karacorlu M, Karacorlu SA. Serous detachment of macula in cystoid macular edema associated with latanoprost. Eur J Ophthalmol. 2008;18:1014-6.

9. Agange N, Mosaed S. Prostaglandin-induced cystoid macular edema following routine cataract extraction. J Ophthalmol. 2010;2010:690707.

10. Panteleontidis V, Detorakis ET, Pallikaris IG, Tsilimbaris MK. Latanoprostdependent cystoid macular edema following uncomplicated cataract surgery in pseudoexfoliative eyes. Ophthalmic Surg Lasers Imaging. 2010;. doi:10.3928/15428877-20100215-91. 
11. Sacchi M, Villani E, Gilardoni F, Nucci P. Efficacy of intravitreal dexamethasone implant for prostaglandin-induced refractory pseudophakic cystoidmacular edema: case report and review of the literature. Clin Ophthalmol. 2014;8:1253-7.

12. Miyake K, Ota I, Maekubo K, Ichihashi S, Miyake S. Latanoprost accelerates disruption of the blood-aqueous barrier and the incidence of angiographic cystoid macular edema in early postoperative pseudophakias. Arch Ophthalmol. 1999;117:34-40.

13. Küchle M, Nguyen $N$, Hannappel E. The blood-aqueous barrier in eyes with pseudoexfoliation syndrome. Ophthalmic Res. 1995;27:S136-42.
14. Menchini U, Bandello F, Brancato R, Camesasca Fl, Galdini M. Cystoid macular oedema after extracapsular cataract extraction and intraocular lens implantation in diabetic patients without retinopathy. Br J Ophthalmol. 1993;77:208-11.

15. Miyake K, Ibaraki N. Prostaglandins and cystoid macular edema. Surv Ophthalmol. 2002;47:203-18.

16. Davidovici BB, Wolf R. The challenge of drug-rechallenge: facts and controversies. Clin Dermatol. 2010;28:349-53.

\section{Submit your next manuscript to BioMed Central and we will help you at every step:}

- We accept pre-submission inquiries

- Our selector tool helps you to find the most relevant journal

- We provide round the clock customer support

- Convenient online submission

- Thorough peer review

- Inclusion in PubMed and all major indexing services

- Maximum visibility for your research

Submit your manuscript at

www.biomedcentral com/submit 